\title{
Diphtheria Toxoid/Tetanus Toxoid/Inactivated Pertussis Vaccine
}

National Cancer Institute

\section{Source}

National Cancer Institute. Diphtheria Toxoid/Tetanus Toxoid/Inactivated Pertussis

Vaccine. NCI Thesaurus. Code C97122.

A vaccine containing detoxified tetanus toxoid, detoxified diphtheria toxoid and inactivated pertussis antigens. 